\title{
Emancipatory reflection on a nursing practice-based ethical issue about nurses' paternalistic decision-making for patients
}

Discussion

Lian-Lian Tang*

Department of Nursing Administration, The First Hospital of Qinhuangdao, Qinhuangdao, Hebei 066000, China

Received: 21 October 2018; Accepted: 22 December 2018; Published: 20 March 2019

Abstract: Objective: This study aimed to demonstrate and promote the skill of critical emancipatory reflection through reflecting on a nursing practice-based ethical issue about nurses' paternalistic decision-making for patients. Meanwhile, critical awareness will be developed and the underlying issues of paternalism in nursing decision-making will be analyzed. Then, by applying the procedure, improvement in nursing decision-making practice will be expected.

Methods: Taylor's model of emancipatory reflection with four steps, including construction, deconstruction, confrontation, and reconstruction, is utilized to guide the author's reflection.

Results: Guided by the socialization theory, the author's personal and professional socialization is seen to be associated with the formation of the value of paternalism. The theory of reflexivity is applied to unearth the related issues, including deeper personal value, work environment, as well as historical and cultural contexts. Moreover, the power derived from policy, work relationship, and nursing administration, which could induce paternalism in the author's nursing decision-making practice, was critically debated using the hegemony theory. Finally, new insights into paternalism will be achieved, which enable change in terms of how to facilitate patients' autonomous decision-making.

Conclusions: The process of refection makes it clear that respecting patients' right and performing patient-centered caring are the bases to change the paternalism existing in the nursing decision-making practice currently. The reconstruction step assists the author in terms of how to value the patients' autonomy and balance patients' safety and choice, rather than being overprotective; carry out risk assessment, and search for strong evidence to counterbalance the positive and negative aspects of risk-taking; communicate with patients appropriately in a manner that they can comprehend; spend more time to explore patients' preference and choice; make every effort to elevate the patients' decision-making capacity; implement patient-centered care and shared decision-making in nursing practice; consult with other colleagues and obtain the required support when limitations or challenges exist; try to justify and avoid hidden paternalism behind policy or guidelines; deal with the power in hand well and fairly; and also positively face the powers that constrain the author.

Keywords: reflection • paternalism • critical emancipatory reflection • Taylor's model • decision-making • nursing ethical issue (c) Shanxi Medical Periodical Press.

How to cite this article: Tang LL. Emancipatory reflection on a nursing practice-based ethical issue about nurses' paternalistic decision-making for patients. Front Nurs. 2019; 1: xx-xx.

* Corresponding author.

E-mail: 44452020@qq.com (L. - L. Tang).

¿ Open Access. ๑ 2019 Tang, published by Sciendo. (cc)BY-NC-ND This work is licensed under the Creative Commons Attribution NonCommercial-NoDerivatives 4.0 License. 


\section{Introduction}

This paper arose from the experience of the author, a Chinese nurse who studied in Ireland and experienced the clash between Oriental and Occidental nursing assumptions. In Irish nursing education and practice, critical reflection has burgeoned over a few years. ${ }^{1}$ It has been recognized as a theoretical method and process for analyzing and transforming practice. ${ }^{2}$ Meanwhile, in the module of Ethics, the author encountered a fresh phrase "paternalism in nursing decision-making", which has never been recorded in her nursing practice before. Then, a considerable amount of thinking and research were undertaken by the author. When the author comprehended the related connotation, she was shocked by her previous paternalistic style of decision-making, which actually breached patients' autonomy in work but was rationalized by her thoughts of guaranteeing patients' safety. Therefore, the author utilized the skill of critical reflection to reflect on this widespread ethical issue in Chinese health care but with little attention by Chinese nurses.

Paternalistic nursing practices, wherein nurses confer a treatment or service upon a person without his/her consent, ostensibly due to reasons of limited autonomy or diminished capacity, are widespread in the healthcare setting. ${ }^{3}$ Numerous issues surround it. In this study, the author examines these issues through the medium of reflective writing and discussion.

\section{Methods}

The whole process of this reflection and writing will be generally guided by Taylor's model of emancipatory reflection with four steps, including construction, deconstruction, confrontation, and reconstruction. ${ }^{4}$ First, in the phase of construction, the author captures the essence of paternalistic decision-making in nursing practice. Then, she deconstructs it as a detached observer through review of the related values and beliefs. The theories of socialization, ${ }^{5}$ reflexivity, ${ }^{6}$ and hegemony ${ }^{7-9}$ are adopted to underpin the reflection and help to understand her individual practice, social context, and relationship between both. In the third phase, a critical view is taken to confront and challenge the author's own basic assumptions, even those of the society. Eventually, a new awareness is reconstructed and in the light of the remolded assumption, how to change the practice in future is clear.

\section{Results}

\subsection{Describe}

I have been a qualified nurse for 11 years in a tertiary hospital in China. I had never realized my paternalistic behavior in nursing practice until I learned the module on ethics during my Irish course on Master of Science in Nursing (MSN). In my previous experience, it was ingrained in my mind that the responsibility for ensuring patients' treatment and recovering their health is far more important than respecting patients' autonomy. I was so overprotective that I implemented some selfrighteous safety measures on clients under the condition of interference with their liberty or autonomy. When I learned that my action is a pattern of paternalistic conduct in health-care setting ${ }^{10}$ and that paternalism is an ethical concept contrary to autonomy ${ }^{11}$ and is criticized by scholars, I was shocked. I perceived that my previous nursing service was filled with paternalistic behaviors. For instance, I traditionally put bedrails on the patients' beds without asking for their consent; , several studies even demonstrated that having the side rails up could not reduce falls. ${ }^{12,13}$ The decision was only implemented due to my own thought that it might prevent them from the potential harm of a fall, although the incidence was identified to be very low in numerous research works. I had tried my best to persuade patients to accept some treatment even when they were not happy or could not afford it. I did not respect patients' autonomy well.

\subsection{Inform}

According to Taylor's guidance, I stood away from my action and looked at myself with the eyes of an interested observer to look for the stimuli ${ }^{4}$ that pushed me to act with paternalism. It seemed that I acted in a paternal role due to my belief that nurses are virtuous and have authority over the patients in the ward.$^{14} \mathrm{I}$ might consider that the patients are not wise in the medical field and that they often do not know what is right or which is the better choice. From this viewpoint, I felt that whatever I did for the patients from my professional experience and personal goodwill was in their best interests. I took it for granted that patients should accept my decisions. Moreover, due to my heavy workload, the time-consuming process of communication to obtain their opinion was not considered essential or it could be overlooked.

Additionally, I realized that I took the view that science came first, rather than ethics, in the health-care industry. I believed that health-care professionals should provide service according to scientific guidelines first. As for ethical principles, they are just largely a formality. This opinion also showed that I hold the diseaseoriented nursing idea. I did not look at the patient as a whole person but as a disease. Thinking deeply, I consider that my nursing work was restricted by many social factors. There may be some power to force me to act paternalistic to some extent. 


\subsection{Confront}

I was aware that the formation of the paternalism value was associated with my socialization process, in which family was an undoubtedly essential factor. ${ }^{15}$ I think it has been embedded in my head when I was a child. From my childhood to adolescence, I followed my father's advice without any disobedience. In my eyes, my father had more knowledge and life experience, and I believed his decision for me was wise and beneficial. ${ }^{16}$ I had to respect and accept the decision that my father made for me, even if sometimes I was not happy. Moreover, in memories of my childhood, when I was ill, my family members and I obeyed all the orders of the nurse without any doubts. Once I was a patient in a hospital, I respected and complied with the words of the nurses devoutly. Thus, I was socialized in the early of my life that in a relationship, the person who is more knowledgeable has the power of making the decision for others, while whoever is less knowledgeable should obey the more knowledgeable ones. ${ }^{17}$ It was embedded in my mind that the decision-making right of those who are considered unwise would be unappreciated. Similarly, I extended to my work the belief that patients should accept the orders and decisions made by nurses who have more professional knowledge and experience than them.

Moreover, education was another prominent attributable factor for the formation of paternalism in my professional socialization. ${ }^{15}$ Socialization is a process whereby I learn to behave in accordance with social traditions and mores. ${ }^{15}$ When I studied nursing in the medical college, nursing was regarded as a subject of science regardless of its philosophical nature. The modules related to science, such as biophysics, biochemistry, anatomy, and so on, comprised our main lessons; no subjects related to nursing ethics or philosophy were included, which would enlighten me and modify my professional ethical values. ${ }^{18}$ I was socialized in the education paradigm that science has more power than humanity and philosophy ${ }^{19}$ in the nursing field and that nursing decision and intervention should be determined by science first rather than by feelings or wishes. ${ }^{20,21}$ Professional education did not guide me to respect service users' preferences and choices. Conversely, it provided paternalism a scientific mask and made me neglect the clients' feelings and wishes in my practice. ${ }^{3}$

Furthermore, my work experience, especially, the influence of my workplace, played a very important role in the process of my professional socialization ${ }^{5}$ to form a paternalistic attitude. According to Rajendra and Rachana, ${ }^{15}$ people can have an impact on each other through imitation, suggestion, and sympathy. Paternalism is very popular in the Chinese health-care setting. ${ }^{22}$
In my workplace, most nursing work is performed in accordance with the nursing routine, such as using bedrails for every patient in the intensive care unit (ICU). ${ }^{3}$ The nursing plan was usually made by the nurses without the participation of patients. ${ }^{23}$ These routine actions often resulted in my neglecting the consent of patients. Moreover, owing to the limited nursing human resource, nurses are very busy at work. I would not like to spend time on communicating with patients and their families the available options to ensure that their decisions are informed. Obviously, the work of talking with patients cannot result in any direct benefits in terms of economy for the hospital. Less-informed consent work can save time cost and reduce my workload to some extent, which can promote my efficiency and earn more money for the employer. ${ }^{24}$ The climate of the hospital not only fostered my paternalism assumption but also forced me to execute it.

I was aware that my disease-centered value induced my paternalistic action. The theory of reflexivity ${ }^{25-27}$ provided the evidence that my paternalistic action was associated with my personal value. Disease-centered value seemed to be the strongest belief in my mind. The disease-centered belief resulted in the situation that more attention was paid to patients' health recovery and safety guarantee rather than to patients' autonomy. ${ }^{28,29}$ I have never facilitated patients' choices when some limitation existed: for instance, if I judged that the patient was not very conscious, I often gave some safety measures directly without performing additional assessment or asking his/her opinion. I was overprotective in my nursing work rather than balancing patients' rights, risks, and the responsibility for both. Any strategies that are able to better cure the disease and protect the patients' safety will be adopted and the patients' opinion to disagree remains enfeebled. ${ }^{30}$ Although the organization advocates patient-centered care ${ }^{31}$ the effective implementation of measures that ensure that this value is actualized was not carried out. The busyness of nursing work and a shortage of nursing staff ${ }^{32}$ made me struggle to finish my tasks, with little energy remaining to make an effort to promote patients' decision-making capacity. Moreover, because of my introverted personality, I was not good at communication with others. I realized that I did not communicate appropriately with clients in a manner that they can understand and explore what is important for them, to promote their preference. ${ }^{30}$

With regard to my historical and cultural contexts, both the Chinese traditional medicine history and international nursing history played an essential role in the existence of my nursing paternalism. It is evident that modern health-care ethics in China is greatly influenced by Chinese traditional medical morality, which has a deep tradition of paternalism. ${ }^{16}$ Paternalism in modern 
Chinese health care is the product of humanity of the Confucian ethics, from which two core typical paternalism ideas are inherited. ${ }^{16}$ Firstly, health-care providers should act considering the patients' best interests and decide on behalf of the patients' benefits completely; secondly, health-care providers could make a decision for the patient ignoring or rejecting the autonomy of the patient. Chinese traditional medical ethics emphasizes "the healer parents heart". ${ }^{16}$ These ideas affect modern health-care philosophy and support paternalism in health-related decision-making. Likewise, some clues of paternalism also can be found in the history of nursing theory, which was once used to guide nursing practice. Peplau $^{33}$ originally analogized the nurse as a surrogate mother and the patient as an infant or child or adolescent, with the relationship between nurses and patients expectantly leading the patient to become an adult person. These deep historical influences prevented me from realizing what paternalism is and whether there is something wrong in acting as I did..$^{34}$ Conversely, I took it for granted in my nursing practice; I even considered that it was a good type of action and that I am a virtuous nurse in terms of protecting patients.

I also noticed that the national culture affected not only my belief but also patients' attitude to paternalism in health-related decision-making. ${ }^{11}$ In turn, patients' attitude in the decision-making process could further affect my working manners and values. ${ }^{35}$ The cultures of different countries shape different people's preferences regarding their role in health-related decisionmaking. ${ }^{36-38}$ In the United States, most patients preferred to be given complete information and be involved in the decision-making process. ${ }^{37,39,40}$ Only $10 \%-20 \%$ of patients did not like to know the details of the decision ${ }^{41}$ and $9 \%-17 \%$ preferred to leave decisions to their family or medical professionals..$^{37,42}$ Nevertheless, studies among African-American, Hispanic, and Asian respondents reported more frequently that they preferred the professionals to make the final decisions. ${ }^{37,42}$ A survey in Taiwan ${ }^{43}$ demonstrated that $92.6 \%$ of patients did not make health-related decisions by themselves. This attitude showed that the style of health-related decisionmaking cannot be entirely patient autonomous. It is well known that paternalism is a widely refused ethical principle in health-care decision-making, especially in Western countries, but Chinese patients prefer to leave the decision-making to medical professionals..$^{43}$ This situation enables the survival and acceptance of paternalism, and further, paternalism becomes natural for nurses (e.g., me) in nursing practice for a long period.

Besides socialization and reflexivity, I also noticed that my paternalistic action was associated with my experience of power. ${ }^{7}$ I worked in a power hierarchy that shaped my paternalism. As a nurse, on the one side, my action was under the power of policy, rules, and management, as well as under doctors. On the other side, I also have some power, which is related to the ability to care for patients, ${ }^{44}$ to influence doctors and patients, to control or have autonomy, ${ }^{45,46}$ and to use health-care resources. ${ }^{47}$ The following will show how these kinds of power interacted with each other and shaped my paternalism.

First, my nursing practice was guided by many kinds of knowledge and rules, including medical science and technology, institutional policies, as well as professional standards, guidelines, and so on. Although it is purported that these rules are primarily guided by scientific principles, such principles are usually generated and disseminated by the health-care institutes themselves. ${ }^{3}$ Therefore, these principles cannot keep off the suspicion of paternalism, ${ }^{48}$ which is the way of self-interest for health-care workers with least resistance from the patients. $^{3}$ Moreover, health-care workers, including nurses, routinely conform to various scientific standards and guidelines to provide treatment to patients, with little care about their actual willingness. Breaching of these standards and guidelines by professionals and regulators without considering the patients' willingness is recognized as erroneous. As Hofmann said, paternalism may be hidden behind the veil of technology and science. ${ }^{48}$

Secondly, power was also involved in the relationship between patients and me. ${ }^{49}$ It is apparent that medical knowledge, nursing experience, and skills made me have authority in the nursing field. ${ }^{50,51}$ The psychology of common people is obedience to authority because of the patriarchal system..$^{52}$ Additionally, in China, nurses have a second-high reputation and status, only behind doctors in the hospital. People trust nurses and believe it would not be wrong to conform to nurses' decision in any situation. ${ }^{53}$ Meanwhile, patients who want very much to get along well with doctors often need me to help them connect. As they reached for me, the patients often complied with my paternalistic decision without any complaints. ${ }^{54}$ Moreover, it is true that I controlled some health-care resources, such as some health information and allocation of my own caring time..$^{55}$ These powers give more fertile ground for my paternalism. The patients often chose silence even when I was paternalistic to them ${ }^{56}$ because they were afraid that my revenge might make me allocate less resources for them. Furthermore, with increase in my professional knowledge and experience, I was increasingly confident with my authority in the nursing field. ${ }^{44}$ This resulted in intensification of my paternalism to some extent.

Thirdly, I also realized that the paternalism in my nursing decision-making cannot be separated from the doctor-nurse power relationships ${ }^{54,57}$ as nurses play a 
mediating role between doctors and patients. ${ }^{52}$ In the power hierarchy in a hospital, it is certain that the grade of doctors is higher than that of nurses. ${ }^{58}$ The preferment or nurses' need for doctors' comments determined that some actions of mine did not consider patients' opinion completely but aimed for a good relationship with or appraisal from doctors. For example, when I executed a doctor's medicine orders, if the patients expressed some disagreement with the order, my dealing habitude was to try my best or utilize my power to make the patients accept the treatment, which is a pattern of paternalism, ${ }^{3}$ instead of asking the doctor to come and explain clearly, because asking the doctor to explain or change the order is not a wise or effective conduct in doctors' eyes. Doctors expected me to deal with these "troubles" for them. Moreover, under the power of doctors, I had never questioned the paternalism involved in the doctors' orders but performed them blindly.

Finally, I felt that the power of nursing management in me also increased my paternalism. ${ }^{59,60}$ As Fook, ${ }^{61}$ Agger, ${ }^{62}$ and Oudshoorn ${ }^{63}$ stated, personal power interacts with the structural level of power. - My action was constrained by the nursing management system. ${ }^{63,64}$ The evaluation system for efficiency, which was invented by the management department of my hospital, put the amount of practical nursing tasks first and foremost. In the context of the Chinese market-oriented economy, the amount of tasks and the profits that I produced determined my income and promotion. ${ }^{65}$ The nursing personnel were also assigned according to the amount of practical nursing tasks, ${ }^{66}$ such as injection, infusion, urine catheterization, and so on. The process of communication with patients and the informing duty had never been taken into account in the workload of nurses. Besides, they were indeed difficult to be calculated and assessed. The root cause was that those nursing operations that could generate economic benefits for the hospital while respecting the patients' rights could not produce immediate profits. With regard to evaluating the work of respecting patients' autonomy in nursing decision-making, ${ }^{67}$ the patient's signature on the informed consent form is the main criterion and evidence used by the manager. This management style causes me to attach greater importance to practical tasks; I was not willing and did not have much time to provide plenty of information to patients and wait for their decision. In contrast, choosing to inform the patients of my decision directly and gaining their signatures was a better way for me to satisfy the requirement of the manager.

\subsection{Reconstruct}

It seemed paternalism in nursing decision-making saved some working time for me, but now I realize that it actually made me take on more responsibilities ${ }^{68,69}$ for the patients' care beyond the call of my duty. ${ }^{10,70}$ Although I tried my best to make a right - and the best - decision for patients, if there was something wrong associated with this decision, the patients and the management would blame me and ask me to face the consequence for the errors. ${ }^{71}$ Hence, I should be responsible for all the negative results generated from the decision that I made for the patients. ${ }^{71}$ Previously, I even thought that it was indeed my responsibility as it was caused by my inappropriate decision, but now I know that my thought was wrong. The root reason that they asked me to take the responsibility was that the decision was not made by the patients themselves and my paternalism deprived patients' autonomy at the time of decision-making..$^{10}$ If the decision had originated from the patients' autonomy completely, they were willing to afford and bear by themselves any consequences arising due to their own decisions. ${ }^{69}$ Therefore, I realized that paternalism not only is against patients' autonomy but also brings forth nursing disputes. ${ }^{72}$

The understanding of paternalism in nursing decision-making is significant for me as a nursing practitioner because it led me to explore my outdated values and realize the necessity of transforming it. As the next step, I will need to reconstruct the understanding with my increased awareness. ${ }^{4}$ I will value the patients' autonomy and respect their knowledge. I am willing to balance patients' safety and choice rather than being overprotective; appreciate patients' right to make decisions; carry out risk assessment and search for strong evidence to balance the positive and negative aspects of risk-taking; communicate with patients appropriately in a manner that they can comprehend; spend more time to explore patients' preference and choice; make every effort to elevate the patients' decision-making capacity; when I am unable to support a patient's decision, explain clearly the reasons to make them understand; implement patient-centered care and shared decision-making in nursing; and consult with other colleagues and obtain the required support when limitations or challenges exist. Moreover, I will try my best to justify the hidden paternalism first to avoid it before the utilization of nursing guidelines, routines, and policies. Furthermore, I need to deal with the power in my hand well and fairly, as well as positively face the powers that constrain me.

\section{Discussion}

Critical reflection is a theoretical method and a process for understanding and transforming practice. Using critical incidents, I identified my own assumptions and values, as well as those of my workplace and even 
nation. ${ }^{61}$ A new reconstruction of my practice assumption was developed. This may also lead to changes in the embedded paternalistic value in my organizational culture. Through reflection, a useful ethical guideline that avoids paternalism in my nursing decision-making was developed. Firstly, respecting clients and avoiding prejudging should be regarded as the two antecedents necessary to sustain values and actions for caring. I certify that respecting is the guiding value and the foundation to interact with patients. ${ }^{73}$ Secondly, I should be sensitive and open to the interplay between protective and empowering actions to accomplish my responsibility of ensuring patient safety and autonomy choice. A good way to promote patients' autonomy is to encourage and invite them to participate in the amendment of the daily caring plan that contributes to their convalescence, health recovery, and quality of life. Ethical principles can serve as guides to practice, but reflection is a tool that promotes my sensitivity and openness and assists me in applying these principles in practice.

\section{Conclusions}

Overall, the reflective process on the paternalism in my nursing decision-making, using Taylor's model of emancipatory reflection, transforms my outdated values and awakens me to the prospect of promoting patients' autonomy in decision-making. The theories of socialization, ${ }^{5}$ reflexivity, ${ }^{6}$ and hegemony ${ }^{8}$ underpinned my reflection and guided me to effectively unearth the values involved in connecting with my experience and

\section{References}

1. Finlay L. Reflecting on "reflective practice". PBPL paper 52. 2008.

2. Gardner F. Creating a climate for change: critical reflection and organisations. Organ Collect. 2007;7:73-80.

3. Cody WK. Paternalism in nursing and healthcare: central issues and their relation to theory. Nurs Sci Q. 2003;16:288-296.

4. Taylor BJ. Reflective Practice. New York: Two Penn Plaza. 2006.

5. Young ME, Stuenkel DL, Bawel-Brinkley K. Strategies for easing the role transformation of graduate nurses. J Nurses Staff Dev. 2008;24:105-110; quiz 111-112.

6. Fook J, Gardner F. Practising Critical Reflection: A Resource Handbook. Berkshire: Open University Press. 2007.

7. Alm TG. Power in nursing. J Adv Nurs. 1991;16:503. social context. Moreover, the paternalism in my nursing practice has been recognized to be related to personal value, history, culture, powers in the working environment, and the attitude of patients. I realized that respecting patients' rights and performing patient-centered caring are the bases of nursing practice. Reconstruction assisted me in the following: value patients' autonomy; balance patients' safety and choice rather than being overprotective; carry out risk assessment and search for strong evidence to balance the positive and negative aspects of risk-taking; communicate with patients appropriately in a manner that they can comprehend; spend more time to explore patients' preference and choice; make every effort to elevate the patients' decision-making capacity; when I am unable to support a patient's decision, to clearly explain the reasons and make them understand; implement patient-centered care and shared decision-making in nursing; and consult with other colleagues to obtain the required support when limitations or challenges exist; try my best to justify and avoid hidden paternalism in policy or guidelines; deal with the power in my hand well and fairly; and positively face the powers that constrain me. Furthermore, I also expect that my transformation in terms of recognition of paternalism and avoiding it in practice will have a related impact on the organizational culture of my hospital.

\section{Conflicts of interest}

The author declares no conflicts of interest.

8. Gordon NS, Wimpenny P. A critical reflection on the personal impact of managerial hegemony within nurse education. J Adv Nurs. 1996;23:479-486.

9. Forgacs D. ed. An Antonio Gramsci Reader: Selected Writings 1916-1935. New York: Schocken Books. 1988.

10. Beauchamp TL, Childress JF. Principles of Biomedical Ethics. Oxford: Oxford University Press. 2001.

11. Rodriguez-Osorio CA, Dominguez-Cherit G. Medical decision making: paternalism versus patientcentered (autonomous) care. Curr Opin Crit Care. 2008;14:708-713.

12. Capezuti E, Maislin G, Strumpf N, Evans LK. Side rail use and bed-related fall outcomes among nursing home residents. J Am Geriatr Soc. 2002;50:90-96.

13. Mosley A, Galindo-Ciocon D, Peak N, West MJ. Initiation and evaluation of a research-based fall prevention program. J Nurs Care Qual. 1998;13:38-44. 
14. Chun AHW, Chan SHC, Lam GPS, Tsang FMF, Wong J, Yeung DWM. Nurse rostering at the hospital authority of Hong Kong. Seventeenth National Conference on Artificial Intelligence and Twelfth Conference on Innovative Applications of Artificial Intelligence. AAAI Press. 2000:951-956.

15. Rajendra KS, Rachana S. Social Psychology. Delhi: Mehra Offset Press. 1997.

16. Zhou Y. Research on medical paternalism of Chinese tradition. Hunan Normal University. 2013 (in Chinese).

17. Bruffee KA. Collaborative Learning: Higher Education, Interdependence, and the Authority of Knowledge. New York: Johns Hopkins University Press. 1999.

18. Li CY, Jin JZ, Cui RS. Research on nursing ethics education status in 35 nursing college and university in China. Chin Nurs Manage. 2006;6:64 (in Chinese).

19. Porter R. The Greatest Benefit to Mankind: A Medical History of Humanity (The Norton History of Science). London: WW Norton \& Company. 1999.

20. Smith KV, Godfrey NS. Being a good nurse and doing the right thing: a qualitative study. Nurs Ethics. 2002;9:301-312.

21. Fealy GM. 'The good nurse': visions and values in images of the nurse. J Adv Nurs. 2004;46:649-656.

22. Chen L, Liu J, Liu Y, Sun W. Problems and relevant solutions of medical inform obligation fulfillment in our country. Chin Med Record. 2013;14:15-17 (in Chinese).

23. Hawes C, Morris JN, Phillips CD, Mor V, Fries BE, Nonemaker S. Reliability estimates for the Minimum Data Set for nursing home resident assessment and care screening (MDS). Gerontologist. 1995;35:172-178

24. Timmins F, de Vries JM. Nurses are not bystanders: a response to Paley. Nurse Educ Today. 2014;34:1269.

25. Steier F. ed. Research and Reflexivity. London: Sage. 1991.

26. Fook J. Reflexivity as method. Annu Rev Health Soc Sci. 1999;9:11-20.

27. White SJ. Unsettling Reflections: The Reflexive Practitioner as 'Trickster' in Inter-Professional Work. Maidenhead: Open University Press. 2006.

28. Wang JW, Ma C, Zhang Q, Ji YF, Liang L. Respect and distort: the view on the harmonious doctorpatient relationship from the perspectives of autonomy, medical paternity and their relationship. Med Philos. 2012;12:46-48 (in Chinese).

29. Appelbaum PS, Lidz CW, Meisel A. Informed Consent: Legal Theory and Clinical Practice. Oxford: Oxford University Press. 1987.
30. Chiovitti RF. Theory of protective empowering for balancing patient safety and choices. Nurs Ethics. 2011;18:88-101.

31. Epstein RM, Street RL Jr. The values and value of patient-centered care. Ann Fam Med. 2011;9:100-103.

32. Choi SP, Cheung K, Pang SM. Attributes of nursing work environment as predictors of registered nurses' job satisfaction and intention to leave. $J$ Nurs Manage. 2013;21:429-439.

33. Peplau HE. Interpersonal Relations in Nursing. NewYork: G. P. Putnam's Sons. 1952.

34. Lindholm C. Culture and identity: The History, Theory, and Practice of Psychological Anthropology. New York: Oneworld Publications Limited. 2007.

35. Thompson N. The Critically Reflective Practitioner. Palgrave: Macmillan. 2008.

36. Charles CA, Whelan T, Gafni A, Willan A, Farrell $S$. Shared treatment decision making: what does it mean to physicians? J Clin Oncol. 2003;21:932-936.

37. Murray E, Pollack L, White M, Lo B. Clinical decision-making: physicians' preferences and experiences. BMC Fam Pract. 2007;8:10

38. Levinson W, Kao A, Kuby A, Thisted RA. Not all patients want to participate in decision making. A national study of public preferences. J Gen Intern Med. 2005;20:531-535.

39. Butow PN, Maclean M, Dunn SM, Tattersall MH, Boyer MJ. The dynamics of change: cancer patients' preferences for information, involvement and support. Ann Oncol. 1997;8:857-863.

40. Mazur DJ, Hickam DH. Patients' preferences for risk disclosure and role in decision making for invasive medical procedures. J Gen Intern Med. 1997;12:114-117.

41. Schattner A. What do patients really want to know? QJM. 2002;95:135-136.

42. Sekimoto M, Asai A, Ohnishi M, et al. Patients' preferences for involvement in treatment decision making in Japan. BMC Fam Pract. 2004;5:1.

43. Tai MC, Tsai TP. Who makes the decision? Patient's autonomy vs paternalism in a Confucian society. Croat Med J. 2003;44:558-561.

44. Sepasi RR, Abbaszadeh A, Borhani F, Rafiei H. Nurses' perceptions of the concept of power in nursing: a qualitative research. J Clin Diagn Res. 2016;10:LC10-LC15.

45. Du Plat-Jones J. Power and representation in nursing: a literature review. Nurs Stand. 1999;13:39-42.

46. Chandler GE. The source and process of empowerment. Nurs Adm Q. 1992;16:65-71.

47. Hawks $\mathrm{JH}$. Empowerment in nursing education: concept analysis and application to philosophy, learning and instruction. J Adv Nurs. 1992;17:609-618. 
48. Hofmann B. Technological paternalism: on how medicine has reformed ethics and how technology can refine moral theory. Sci Eng Ethics. 2003;9:343-352.

49. Kettunen T, Poskiparta M, Gerlander M. Nursepatient power relationship: preliminary evidence of patients' power messages. Patient Educ Couns. 2002;47:101-113.

50. Katriina P, Sari V, Anja R, Christina S, Paula A, Tarja S. Nursing power as viewed by nursing professionals. Scand J Caring Sci. 2013;27:580-588.

51. Adib Hagbaghery M, Salsali M, Ahmadi F. A qualitative study of Iranian nurses' understanding and experiences of professional power. Hum Resour Health. 2004;2:9.

52. Cong Y. Doctor-family-patient relationship: the Chinese Paradigm of informed consent. J Med Philos. 2004;29:149-178.

53. Kuokkanen L, Leino-Kilpi $H$. The qualities of an empowered nurse and the factors involved. J Nurs Manage. 2001;9:273-280.

54. Porter S. A participant observation study of power relations between nurses and doctors in a general hospital. J Adv Nurs. 1991;16:728-735.

55. Dolores D, McCarthy J. Nursing Ethics: Irish Cases and Concerns. Dublin: Gill \& Macmillan. 2005.

56. Heineken J. Patient silence is not necessarily client satisfaction: communication problems in home care nursing. Home Healthc Nurse. 1998;16:115-120; quiz 120-121.

57. Keddy B, Gillis MJ, Jacobs P, Burton H, Rogers M. The doctor-nurse relationship: an historical perspective. J Adv Nurs. 1986;11:745-753.

58. Reeves S, Nelson S, Zwarenstein M. The doctornurse game in the age of interprofessional care: a view from Canada. Nurs Inq. 2008;15:1-2.

59. Manojlovich M. Promoting nurses' self-efficacy: a leadership strategy to improve practice. J Nurs Admin. 2005;35:271-278.
60. Antrobus S, Kitson A. Nursing leadership: influencing and shaping health policy and nursing practice. J Adv Nurs. 1999;29:746-753.

61. Fook J. Social Work Critical Theory and Practice. London: Sage. 2002.

62. Agger B. Critical Social Theories. Boulder/Oxford: Westview Press. 1998.

63. Oudshoorn A. Power and empowerment: critical concepts in the nurse-client relationship. Contemp Nurse. 2005;20:57-66.

64. Tomey AM. Nursing Management and Leadership. London: Sage. 2009.

65. Keister LA. Adapting to radical change: strategy and environment in piece-rate adoption during China's transition. Organ Sci. 2016;13:459-474.

66. Zhang LD, Yang G. The management of nursing human resource. Health Nutr China. 2013;6:15171518 (in Chinese).

67. Lewis FM, Batey MV. Clarifying autonomy and accountability in nursing service: part 2. J Nurs Adm. 1982;12:10-15.

68. Aycan Z. Indigenous and Cultural Psychology. Istanbul: Springer US. 2006.

69. Coulter A. Paternalism or partnership? Patients have grown up-and there's no going back. BMJ. 1999;319:719-720.

70. Cunliffe P. Dangerous duties: power, paternalism and the "responsibility to protect". Rev Int Stud. 2010;36:79-96.

71. Hart HC. Anarchy, paternalism, or collective responsibility under the canals? Economic Political Weekly. 1978;13:A125-A134.

72. Mckeown M. Alliances in action: opportunities and threats to solidarity between workers and service users in health and social care disputes. Soc Theory Health. 2009;7:148-169.

73. Chiovitti RF. Nurses' meaning of caring with patients in acute psychiatric hospital settings: a grounded theory study. Int J Nurs Stud. 2008;45:203-223. 